\title{
Avoidance decrement: Replication and further analysis'
}

\author{
James H. Reynierse, ${ }^{2}$ Dominie J. Zerbolio and M. Ray Denny
} MICHIGAN STATE UNIVERSITY

\begin{abstract}
Twenty Sprague-Dawley rats, 8 females and 12 males, were run 100 trials with a buzzer or tone CS for 10 consecutive days in a wheel turn avoidance apparatus. About $50 \%$ of the Ss showed a decrement in avoidance performance with continued training. CS and sex variables did not systematically affect the decrement phenomenon. Non-decrementing Ss displayed higher avoidance performance then decrementers both within and between sessions. No significant difference in warmup magnitude between decrementers and non-decrementers was found. These data are interpreted as indicating the learning of a competing CER (freeze) response to the CS which may produce the decrement phenomenon.

\section{Introduction}

Several recent studies using similar techniques, have found a decrease in avoidance responding with continued training (Coons, Anderson \& Meyers, 1960; Smith, McFarland \& Taylor, 1961; and Anderson \& Nakamura, 1964). These studies used a wheel turn avoidance apparatus and a discriminated avoidance procedure. The present study replicates this procedure and examines the results in greater detail.

\section{Subjects}

The Ss were 20 experimentally naive albino rats (8 females and 12 males) 120-150 days old from the colony maintained by the Psychology Department at Michigan State University. All Ss were Sprague-Dawley derivatives.

\section{Apparatus}

All Ss were run in two identical wheel turn chambers with CS, US, and responses monitored by electrical programming equipment and appropriate relay circuitry. The apparatus is described in detail elsewhere (Zerbolio, Reynierse, Weisman \& Denny, in press).

\section{Procedure}

All Ss were given 100 trials per day for 10 consecutive days. Ten Ss ( 4 females and 6 males) had a buzzer CS (Malis \& Curran, 1960)paired with $1.0 \mathrm{ma}$ shock and delivered to the grid floors by Grason Stadler shock scramblers, Model E1064GS. Ten Ss (4 females and 6 males) had a $4000 \mathrm{cps}$ pure tone CS. The CS-US interval was $5 \mathrm{sec}$. and both CS and US were response terminated when $S$ made a $1 / 4$ wheel turn. A variable intertrial interval with a mean of $80 \mathrm{sec}$. and ranging from 40-120 sec. was used.

\section{Results}

The number of avoidances per day for each $\mathrm{S}$ was plotted for 10 consecutive days. Tabulation of the data in this manner suggested that there were two distinct classes of Ss, decrementers and non-decrementers. Since asymptotic performance or peak performance was reached by all Ss on or before Day 3, decrement was defined as follows. If an Ss' avoidance level dropped to $50 \%$ or less of his best performance on the first three running days and stabilized at or below this level, decrement was considered to have occurred. Using this criterion, Ss were divided into decrementers or nondecrementers. One S (tone CS male) made a total of 15 avoidances over the 10 sessions and was eliminated from all analyses for failure to learn. Chi Square tests for the effects of CS and sex of S on producing decrement were not significant ( $x 2=.493$ and .073 respectively); therefore decrementers and non-decrementers were grouped independently of CS and sex variables.

Figure 1 presents the mean avoidances and wheel turns for decrementers $(\mathrm{N}=9)$ and non-decrementers $(\mathrm{N}=10)$ over 10 consecutive sessions. The non-decrementers stabilized at approximately $80 \%$ avoidances by Day 2. But the decrementers reached a peak by Day 2 and then displayed progressively deteriorating performance with additional sessions. Differences between decrementers' and non-decrementers' avoidance levels reached statistical significance by Day $8(t=3.023$; $\mathrm{df}=9 ; \mathrm{p}<.02$ ) and remained significantly different thereafter although the trend appeared much earlier.

Wheel turn rates for every $\mathrm{S}$, independent of number of avoidances, tended to decline with successive ses-

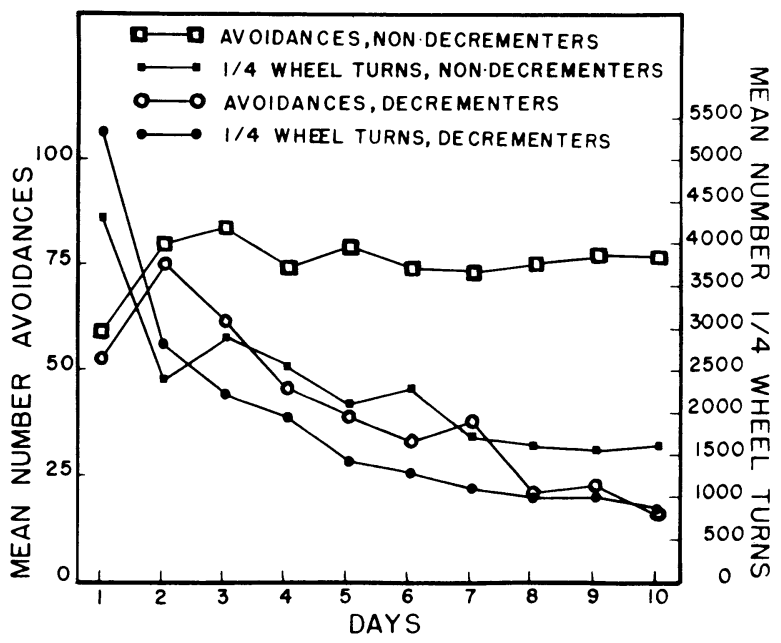

Fig. 1. Mean avoidances and mean $1 / 4$ wheel turn rates per day for decrementers and non-decrementers for 10 consecutive days. 


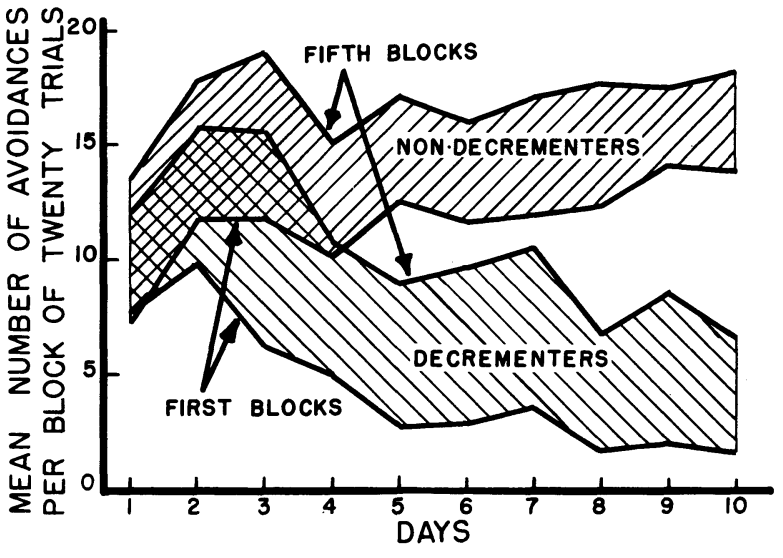

Fig. 2. Warm-up effect for decrementers and non-decrementers for 10 consecutive days. The lower and upper bounds for decrementers and non-decrementers represent the mean avoidances per day for the first and fift' blocks of 20 trials respectively.

sions. Initially wheel turn rates for decrementers and non-decrementers did not differ statistically. But, by Day 8, non-decrementers showed significantly more wheel turns than decrementers $(t=2.496 ; d f=9 ; p<.05)$ and this difference remained on subsequent sessions.

An obviously significant warm-up effect was noted in both groups over all 10 sessions. Figure 2 presents the mean avoidances for the first and last blocks of 20 trials each day for both groups. The middle three blocks are not presented, as they yielded intermediate performance for both groups. The mean warm-up (the difference between the first and fifth blocks over 10 days) was not significantly different for the two groups $(\mathrm{t}=.102)$.

\section{Discussion}

The present study confirms the avoidance decrement phenomenon that has been reported previously. But a detailed analysis of the effect reveals that only some Ss (about $50 \%$ in the present study) show the effect.

Neither sex of S nor quality of CS selectively produced the decrement although Anderson \& Nakamura (1964) have previously reported sex differences. An analysis of the observed warm-up effect indicates that improvement within a session is comparable for decrementers and non-decrementers. But the absolute level of avoidance for decrementers is markedly lower at every point within a session. On this basis it appears that the decrement phenomenon is a generalized effect which influences performance throughout a session.

Behavioral observations suggest that, with continued training, decrementers show an increased tendency to freeze (CER) during CS presentations. This increasing tendency to freeze may represent the learning of a response that is incompatible with the avoidance response and, by effectively competing with it, could account for the decrement phenomenon. Such an explanation is consistent with the finding that the wheel turn rates of decrementers is markedly lower than that of non-decrementers.

\section{heferences}

ANDERSON, N. H., \& NAKAMURA, C. Y. Avoidance decrement in avoidance conditioning. J. comp. physiol. Psychol., 1964, 57, 196-204.

COONS, E. E., ANDERSON, N. H., \& MYERS, A. K. Disappearance of avoidance behavior during continued training. J. comp. physiol. Psychol., 1960, 53, 290-292.

MALIS, J. L., \& CURRAN, C. S. A reliable, low-cost generator for audio stimuli. J. exp. Anal. Behav., 1960, 3, 200.

SMITH, O. A., McFARLAND, W. L., \& TAYLOR, E. Performance in a shock-avoidance conditioning situation: interpreted as pseudoconditioning. J. comp. physiol. Psychol., 1961, 54, 154-157.

ZERBOLIO, D. J., REYNIERSE, J. H., WEISMAN, R. G., \& DENNY, M. R. Pseudoconditioning? J. comp. physiol. Psychol., in press.

\section{Notes}

1. This work was supported by a National Science Foundation Research Grant, BE-238, awarded to M. Ray Denny.

2. Now at Indiana University. 